\title{
Analysis of changes in blood flow oscillations under different probe pressure using laser Doppler spectrum decomposition
}

Igor O. Kozlov, Evgeny A. Zherebtsov, Mikhail A. Mezentsev, Valery V. Shupletsov, Elena V. Potapova, et al.

Igor O. Kozlov, Evgeny A. Zherebtsov, Mikhail A. Mezentsev, Valery V. Shupletsov, Elena V. Potapova, Angelina I. Zherebtsova, Viktor V. Dremin, Andrey V. Dunaev, Igor V. Meglinski, "Analysis of changes in blood flow oscillations under different probe pressure using laser Doppler spectrum decomposition," Proc. SPIE 11079, Medical Laser Applications and LaserTissue Interactions IX, 110791Q (22 July 2019); doi: 10.1117/12.2527040 


\title{
Analysis of changes in blood flow oscillations under different probe pressure using laser Doppler spectrum decomposition
}

\author{
Igor O. Kozlova , Evgeny A. Zherebtsova, b, Mikhail A. Mezentseva , Valery V. Shupletsova ${ }^{\mathrm{a}}$, \\ Elena V. Potapova ${ }^{\mathrm{a}}$, Angelina I. Zherebtsova ${ }^{\mathrm{a}}$, Viktor V. Dremin ${ }^{\mathrm{a}, \mathrm{b}}$, Andrey V. Dunaev ${ }^{\mathrm{a}}$, and \\ Igor V. Meglinski ${ }^{\mathrm{b}}$ \\ ${ }^{a}$ Orel State University named after I.S. Turgenev, Komsomolskaya St. 95, Orel, Russia \\ ${ }^{\mathrm{b}}$ Optoelectronics and Measurement Techniques, University of Oulu, Oulu, FI-90014, Finland
}

\begin{abstract}
Presently, in the modern laser Doppler flowmetry (LDF) the distribution of blood perfusion and its changes along the Doppler shift frequencies are simply ignored and/or not properly addressed. Utilizing the registered power spectrum of photocurrent, we introduce an LDF signal processing approach suitable for expanding of diagnostic capabilities of the technique. In particular, we demonstrate that it is possible to determine how the oscillations of blood flow (cardiac, breathe, myogenic, etc.) are distributed along the Doppler shift frequency. Wavelet analysis is utilized to extract the oscillations corresponded to the particular frequency sub-bands of blood perfusion. The main purpose of this study is to identify influence of local pressure by fiber optic probe on cardiac oscillations and their distribution along frequency of Doppler shift.
\end{abstract}

Keywords: Laser Doppler flowmetry, spectrum decomposition, blood flow oscillations, blood perfusion, probe pressure effects

\section{INTRODUCTION}

The study of microcirculation by laser Doppler flowmetry is often used in diagnostics, clinical practice and research. ${ }^{1,2}$ This technique is relatively simple and non-invasive. In addition, it can be done by a wearable device like a smartwatch. ${ }^{3}$ The principle of LDF is registration of back-scattered photons from moving ensembles of red blood cells in the skin. ${ }^{4}$ The measured parameter in this technology is blood perfusion. According to classical research in this field, ${ }^{5}$ blood perfusion is proportional the mean concentration and velocity of red blood cells in diagnostic volume. The diagnostic significance of this parameter is associated with blood flow oscillations $^{6}$ (cardiac, breathe, myogenic, neurogenic and endothelial) and mean level of blood perfusion. A wide range of diseases affects the functionality and structure of microvascular bed. The averaging of the Dopplerbroadening spectrum obtained by conventional laser Doppler flowmetry (cLDF) occurs with loss information about signal distribution. Whereas, saving and integration over the frequency sub-bands can provide details about contribution of these sub-bands in the overall signal. Moreover, it is possible to implement wavelet analysis for receiving the information about oscillation amplitude in every Doppler shift frequency sub-bands. Local pressure from optical probe was chosen as an affecting factor. The various levels of pressure exerted on the microvascular bed had influence on the localisation of blood flow oscillation in frequency sub-bands of Doppler shift. In this study, we analysed the distribution of cardiac oscillations depending on local pressure in group of healthy volunteers.

\section{MATERIAL AND METHOD}

Eight healthy volunteers were involved in our study. Special 3d-printed tooling and set of $30 \mathrm{~g}$ weights were used for various level of local pressure modelling. All experiments were divided into 6 stages: 0 (1 stage), 30 (2 stage), 90 (3 stage), 150 (4 stage), 210 (5 stage), 30 (6 stage) mm Hg. Duration of every stage was 10 minutes. Registration of blood perfusion was performed with self-developed LDF device that consists $1064 \mathrm{~nm}$ one-mode

Further author information: (Send correspondence to Igor O. Kozlov)

Igor O. Kozlov: E-mail: i.kozlov@oreluniver.ru, Telephone: +7 9208140458

Medical Laser Applications and Laser-Tissue Interactions IX, edited by Lothar D. Lilge, Carsten M. Philipp, Proc. of SPIE Vol. 11079, 110791Q · C) 2019 SPIE · CCC code: 1605-7422/19/\$21 · doi: 10.1117/12.2527040 
laser, two photodiodes, AC-DC amplification board and data acquisition board NI USB-6211. Signal processing, data storage and control of the device was carried out on PC with LabVIEW-designed special application. Sampling rate of signal was $50 \mathrm{kS} / \mathrm{s}$. Sampling rate of perfusion measurement was $20 \mathrm{~S} / \mathrm{s}$. Power spectrum was processed and saved from 0 to $12800 \mathrm{~Hz}$. Every spectrum sequence was divided in sub-bands with $200 \mathrm{~Hz}$ step (0-200, $200400 \mathrm{~Hz}$, etc). After that, blood perfusion was calculated according to following equation:

$$
P U=\int_{f_{1}}^{f_{2}} f \cdot S(f) d f,
$$

where $S(f)$ - Doppler shift amplitude of power spectrum on frequency $f ; f_{1}, f_{2}$ - limits of the integration over frequencies of the registered spectrum. ${ }^{5}$ Morlet core function was used for wavelet analysis of received set of blood perfusion records:

$$
\psi(t)=e^{2 \pi i} \cdot e^{-t^{2} / 2 a^{2}}
$$

where $a=1$. Result of signal processing ${ }^{7}$ can be showed as waterfall figure (Fig. 1) .

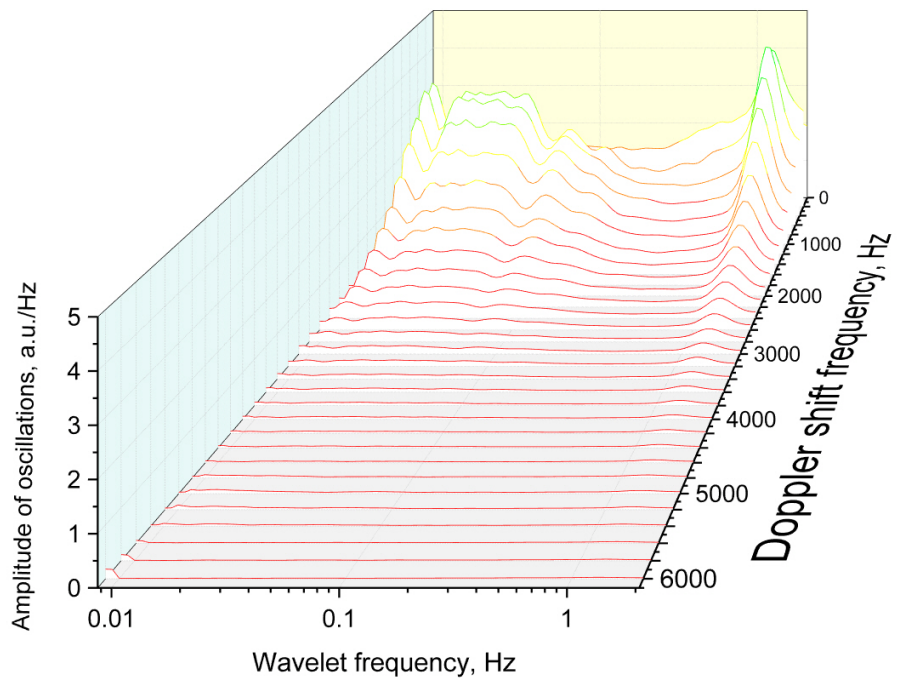

Figure 1. Example of wavelet analysis from every Doppler shift frequency sub-bands of integration

\section{EXPERIMENT RESULTS AND DISCUSSION}

Results of eight processed experiments with distribution of cardiac oscillation respectively frequency of Doppler shift are shown at Fig. 2. In the normal case, large number of Doppler shift sub-bands are modulated by heart with significant amplitude value. However, pressure at the stage $2(30 \mathrm{~mm} \mathrm{Hg})$ and the stage $3(90 \mathrm{~mm}$ $\mathrm{Hg}$ ) provoked further broadening of cardiac oscillation maximum along Doppler shift frequencies. Increasing of pressure (at stages 4 and 5) led to following redistribution of cardiac oscillation amplitude in sub-bands between 200 and $800 \mathrm{~Hz}$ and decreasing in other sub-bands. At the stage $6(30 \mathrm{~mm} \mathrm{Hg})$, returning the maximum of cardiac oscillations to other sub-bands is showed due relaxation of compressed vessels. Phenomenon of oscillation amplitude increasing during stage 2 and stage 3 may be connected with immersing of the probe into the tissue. In this case, diagnostic volume should increase without clamping the arterioles. Thus, additional quantity of oscillating vessels should involved in pulse signal formation. A high amplitude level in Doppler shift frequency sub-bands more than $1000 \mathrm{~Hz}$ during stage 3 may be associated with blood flow in deeper arterioles than it is reached during basal measurements (stage 1). As it shown on Fig. 2d and Fig. 2f, shape of the graphs during stages 4 and 5 has a sharp maximum at low-frequency sub-bands compared with stages 1,2,3 and 6 . As for highfrequency sub-bands, there are low amplitude during stages 4 and 5 at frequencies more than $2000 \mathrm{~Hz}$ compared 


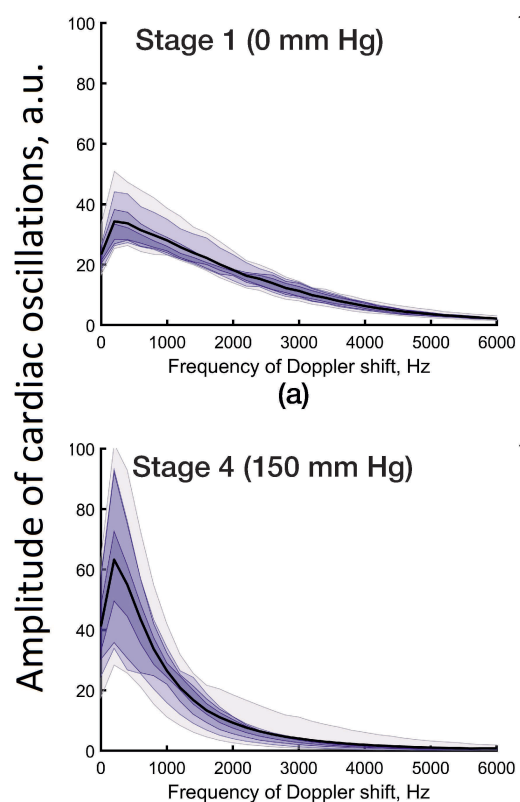

(d)

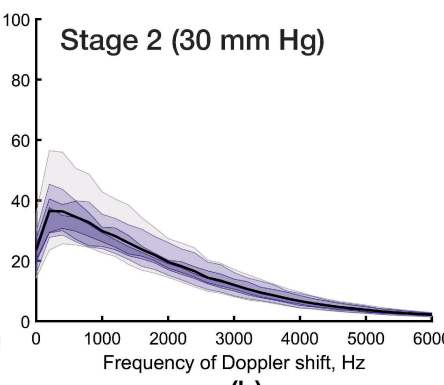

(b)

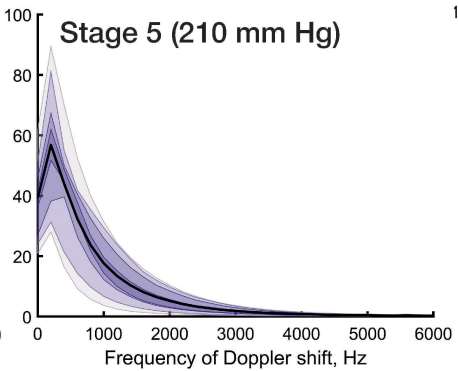

(f)

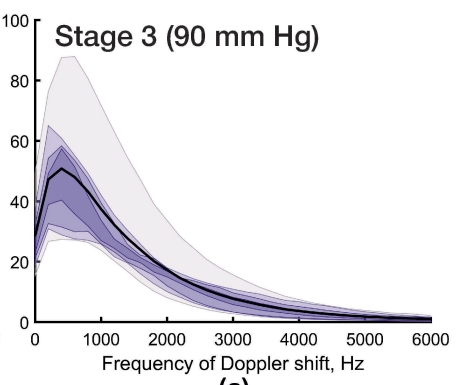

(c)

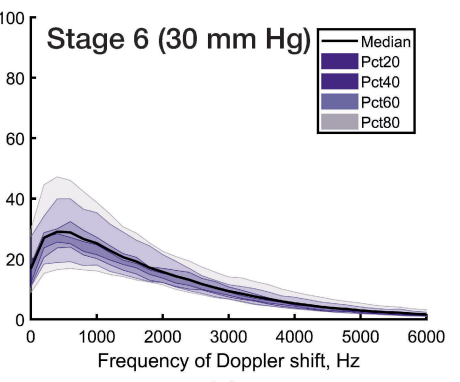

(g)

Figure 2. Amplitude distribution of cardiac oscillation over the frequency of Doppler shift according to stage

with other stages. Lack of amplitudes at this sub-bands during high level of pressure hypothetically occurred due to clamping of capillaries and arterioles. ${ }^{8}$ At the other hand, shear stress in capillaries and small arterioles and venules cause changes on red blood cells aggregation ability that can also affect on laser Doppler spectrum distribution. ${ }^{9}$ The amplitude value growth during stages 4 and 5 at low-frequency sub-bands is associated with the fact that more blood vessels become involved only in cardiac oscillations because of high pressure influence on other oscillations. ${ }^{10}$

\section{CONCLUSIONS}

The study of the Doppler shift frequency distribution of blood perfusion is an interesting and relatively unexplored area as well as the contribution of each Doppler shift frequency band to the microcirculation oscillations. Understanding these processes can shed light on why microcirculation systems make the greatest contribution to the overall perfusion and amplitude of microcirculatory oscillations.

\section{ACKNOWLEDGMENTS}

The work has been funded by the grant of the Russian Science foundation research project 18-79-00237. Evgeny Zherebtsov kindly acknowledges for personal support from grant of Academy of Finland No.318281.

\section{REFERENCES}

[1] Volkov, M., Kostrova, D., Margaryants, N., Gurov, I., N.P., E., Dremin, V., Zharkikh, E., Zherebtsov, E., Kozlov, I., and Dunaev, A., "Evaluation of blood microcirculation parameters by combined use of laser doppler flowmetry and videocapillaroscopy methods," Proc.SPIE 10336, 1033607 (2017).

[2] Zherebtsov, E., Zherebtsova, A., Doronin, A., Dunaev, A., Podmasteryev, K., Bykov, A., and Meglinski, I., "Combined use of laser Doppler flowmetry and skin thermometry for functional diagnostics of intradermal finger vessels," Journal of Biomedical Optics 22(4), 040502 (2017).

[3] Zherebtsov, E., Sokolovski, S., Sidorov, V., Rafailov, I., Dunaev, A., and Rafailov, E. U., "Novel wearable VCSEL-based blood perfusion sensor," 2018 International Conference Laser Optics (ICLO) , 564-564 (June 2018). 
[4] Dunaev, A., Zherebtsov, E., Rogatkin, D., Stewart, N., Sokolovski, S., and Rafailov, E., "Novel measure for the calibration of laser doppler flowmetry devices," Proc.SPIE 8936, 89360D (2014).

[5] Bonner, R. and Nossal, R., "Model for laser Doppler measurements of blood flow in tissue," Applied Optics 20(12), 2097-2107 (1981).

[6] Bračič, M. and Stefanovska, A., "Wavelet-based Analysis of Human Blood-flow Dynamics," Bulletin of Mathematical Biology 60(5), 919-935 (1998).

[7] Kozlov, I., Zherebtsov, E., Dremin, V., Angelina I. Zherebtsova, A., Zharkikh, E., Dunaev, A., and Rafailov, E., "Laser doppler spectrum decomposition applied in diagnostics of microcirculatory disturbances," Proc.SPIE 10685, $106854 \mathrm{O}$ (2018).

[8] Popov, A., Bykov, A., and Meglinski, I., "Influence of probe pressure on diffuse reflectance spectra of human skin measured in vivo," Journal of Biomedical Optics 22, 110504 (2017).

[9] Avsievich, T., Popov, A., Bykov, A., and Meglinski, I., "Mutual interaction of red blood cells assessed by optical tweezers and scanning electron microscopy imaging," Opt. Lett. 43, 3921-3924 (Aug 2018).

[10] Zherebtsov, E., Kandurova, K., Seryogina, E., Kozlov, I., Dremin, V., Zherebtsova, A., Dunaev, A., and Meglinski, I., "The influence of local pressure on evaluation parameters of skin blood perfusion and fluorescence," Proc.SPIE 10336, 1033608 (2017). 